\title{
Comparison of Del Nido and Intermittent Warm Blood Cardioplegia in Adult Coronary Artery Bypass Surgery: Randomized, Prospective, Single Center Trial
}

\author{
Mehmet Acipayam ${ }^{1}$, Erdinc EROGLU ${ }^{1}$, Ahmet Acipayam ${ }^{1}$, Fatih Yazar $^{1}$, Yavuz Orak ${ }^{1}$, \\ and Fatma Tolun ${ }^{1}$ \\ ${ }^{1}$ Kahramanmaras Sutcu Imam University Faculty of Medicine
}

May 28, 2020

\begin{abstract}
Objectives:The purpose ofthis randomized prospective study was to investigate the efficacy and clinical results of del Nido solution (DNS) use in coronary artery bypass grafting (CABG) surgery compared to intermittent warm blood cardioplegia (IWBC). Methods:Thirty-nine adult patients who underwent primary isolated CABG surgery with cardiopulmonary bypass (CPB) between June 2018 and February 2019 were included in the study. DNS was used in 17 patients and IWBC in 22 . The clinical and laboratory results were then compared. Results:Aortic cross-clamp time and CPB time were both lower with DNS. However, we observed no statistically significant difference in clinical results between the two methods, including postoperative myocardial enzymes. Conclusion:Single-dose DNS reduced CPB and aortic cross-clamp times, and provided similar short-term clinical and laboratory outcomes to a conventional multi-dose BC strategy in isolated CABG surgery.
\end{abstract}

\section{Introduction}

Coronary artery bypass is one of the most widely performed adult cardiac surgical procedures. The most frequentlyemployed technique involvescardiopulmonary bypass (CPB) and cardioplegic arrest of the heart.Cardioplegia is a crucial myocardial protection technique for patients from all age groups requiring cardiac surgery necessitating cardiac arrest.A wide range of cardioplegia solutions is currently available for myocardial protection in cardiac surgery. Cardioplegia solutions broadly fall into two main types, based on the basic solutions that comprise the contents of blood and crystalloid cardioplegia. Blood-based cardioplegia solutions bear a close resemblance to normal physiological oxygen transport capacity and appear to be advantageous and involve fewer risks in terms of myocardial protection. In the early 1990s, Dr. Pedro del Nido and his team developed a solution known as "del Nidocardioplegia". This wasoriginally employed for pediatric cardiac surgery, but has recently been employed in a single dose in adult cardiac surgery. del Nidosolution (DNS) can be used in a single dose, yielding a minimum of 90 minutes of cardiac arrest (1).

Only a few retrospective studies have investigated the use of DNScardioplegia in coronary artery bypass in adult patients (2-4). This randomized prospective study was therefore designed to determine the efficacy and safety profiles of DNS compared to IWBC in CABG surgery.

\section{Materials and Methods}

Approval for the study was granted by the local ethical committee, and all participants provided signed written informed consent forms (decision no. 15, session no. 2018/10, dated 16.05.2018). Thirty-nine patients undergoing elective CABG surgery from June 2018 to February 2019 were randomly allocated to one of two groups (DNS being used in 17 patients and IWBC in 22)(Figure 1 ). Patients requiring emergency surgery or any combined operations were excluded. Patients undergoing reoperation for bleeding 
were alsoexcludedsince cardiac enzyme values and myocardial damage cannot beevaluated objectively under such circumstances. All procedures were carried out with CPB and under general anesthesia. Midazolam (ZolamidR, Defarma, Tekirdağ, Turkey) $(0.1 \mathrm{mg} / \mathrm{kg}$ via the intravenous (iv) route), fentanil (TalinatR, Vem, Istanbul, Turkey) (5-8 $\mu \mathrm{g} / \mathrm{kg}$ iv), and rocuronium bromide (MyocronR, Vem, Istanbul, Turkey) (0.6 $\mathrm{mg} / \mathrm{kg}$ iv) were used for anesthesia induction. A Primus device (Drager, Lübeck, Germany) was used for maintenance of general anesthesia, and Sevoflurane (SevoraneR, Abbvie, Istanbul, Turkey) was employed as an intraoperative anesthetic agent.Rocuronium bromide $(0.6 \mathrm{mg} / \mathrm{kg}$ iv $)$ was applied once every $30 \mathrm{~min}$. In addition,320-400 IU/kg unfractionated heparin was administered in order to maintain an activated clotting time (ACT) exceeding $480 \mathrm{sec}$. The ascending aorta was first cannulated, and venous drainage was provided by a single two-stage atrial cannula or bicaval cannulation. A $1600 \mathrm{~mL}$ prime volume $(1500 \mathrm{ml}$ isolate S, $20 \%$ mannitol $100 \mathrm{cc}$, 5000IU heparin) was employed, and 2.0-2.5 L/min $/ \mathrm{m}^{2}$ flow rate, 200-250 $\mathrm{mm} \mathrm{Hg} \mathrm{PaO}_{2}$, and 35-45 mmHg $\mathrm{PCO}_{2}$ were maintained. Blood specimens were collected with the insertion of a retrograde cardioplegia cannula. DNS and IWBC were applied in an antegrade manner. Our myocardial protection routine involves the administration of an additional dose $60 \mathrm{~min}$ after the first dose based on our clinical experienceof DNS. However, none of our patients required a second dose. Following aortic clamping, 1000 $\mathrm{mL}$ DNS was delivered once at $+4^{\circ} \mathrm{C}$. In case of patients receiving IWBC, $15 \mathrm{ml} / \mathrm{kg}$ (maximum $1000 \mathrm{cc}$ ) blood cardioplegia was delivered at $32^{\circ} \mathrm{C}$, and an additional dose was administered every 20 min during the ischemic period. Topical hypothermia was applied in all cases. A membrane oxygenator and an arterial line filter were employed in all cases to maintain a hematocrit (Hct) level $>22 \%$ during $\mathrm{CPB}$, while mean arterial pressure was preserved at 60-80 mmHg.Serum glucose was maintained between 110 and $200 \mathrm{mg} / \mathrm{dL}$ with insulin infusion if required. This was then cooled to $32^{\circ} \mathrm{C}$ (nasopharyngeal core body temperature), and a-stat $\mathrm{pH}$ management was applied.IWBC and DNScardioplegia contents are shown in Table 1.

\section{Laboratory measurements:}

Blood was collected four times in both groups for measurement, using an retrograde cardioplegia cannula -

1. Immediately after entering the cardiopulmonary bypass,

2. 20 min after cross-clampplacement,

3. Immediately before cross-clamp removal, and

4. $20 \mathrm{~min}$ after removal of the cross-clamp. Blood samples were stored at $-80^{\circ} \mathrm{C}$.

Measurement of Serum adenosine deaminase activity: Serum adenosine deaminase activity (ADA) was determined using the spectrophotometric method described byGiuisti, which relies on the indirect calculation of ammonia formation resulting when adenosine deaminase acts in excess of adenosine (5). Results were expressed as units per liter $(\mathrm{U} / \mathrm{L})$.

Thiol/disulfide homeostasis tests: Thiol/disulfide homeostasis was measured with the commercially available novel automatic and spectrophotometric technique developed by Erel and Neselioglu (6) (Rel Assay Diagnostics, Turkey).Using this technique, dynamic and reducible disulfide bonds in the samples were reduced to free functional thiol groups by means of sodium borohydride. In order to avoid unused reduced sodium borohydride being reduced to dithionite-2 nitrobenzoic (DTNB), NaBH4 was removed with formaldehyde. Values for native thiol (NT) and total thiol (TT) were calculated following reaction with DTNB. Disulfide (DS) levels were calculated ashalf the difference of the result obtained by subtracting the native thiol value from the total thiol content.

Measurement of paraoxonase activity: The fully automated method developed by Rel Assay Diagnostics (Mega Tip, Gaziantep, Turkey) was employed to examine serum paraoxonase 1 (PON) activity. This technique involvesparaoxonase activity beingcalculated in medium without $\mathrm{NaCl}$ (basal paraoxonase activity) and with $\mathrm{NaCl}$ (salt-stimulated paraoxonase activity). Hydrolysis of the paraoxone (diethyl-pnitrophenylphosphate) is monitored by observing the increase in absorbance at 37-C and $412 \mathrm{~nm}$. The quantity of p-nitrophenolproduced by the hydrolysis is calculated using the molar absorption coefficient 17,000 Mj1cmj1 (at pH 8). Net values with enzymatic activity are obtained by subtracting the basal activity 
value from the salt-stimulated activity value. The results are expressed as unit per liter, equivalent to the hydrolysis of 1 micromole substrate in one minute and one liter.

Measurement of arylesterase activity: The fully automated method developed by Rel Assay Diagnostics (Mega Tip, Gaziantep, Turkey) was employed to measure paraoxonasearylesterase form as a result ofrase activity in the serum samples. This technique involves the use of phenyl acetate as a substrate for calculatingarylesterase activity. Phenol and acetic acidphenyl acetate hydrolysis. The phenol that forms thenbinds to 4- aminoantipyrine and potassium ferricyanide, and is measured using the colorimetric method. Arylesterase enzyme activity is calculated from $4000 \mathrm{Mj} 1 \mathrm{cmj} 1$, representing the molar absorption coefficient of the colored complex that forms. The results are expressed as unit per liter, equivalent to the hydrolysis of 1 micromole phenyl acetate in one minute and one liter.

Measurement of xanthineoxidase (XOD): XOD measurement was performed using a microELISA kit and aRel Assay DiagnosticsReader device and washer. This was operated manually, with specimens being automatically washed and read.

Measurement of superoxide dismutase (SOD): SOD was measured automatically on a Selectra (The Netherlands) biochemistry autoanalyzer, using aRel Assay Diagnostics automatic biochemistry kit.

Measurement of Creatine kinase-MB (CK-MB): CK-MB was measured automatically using a Rochechemiluminescence method device and a Rochechemiluminescence kit.

Measurement of Troponin I: Troponin I was measured automatically using a Roche chemiluminescence method device and a Roche chemiluminescence kit.

$\mathrm{CPB}$ and cross-clamp times, and lengths of stay in the intensive care unit were recorded. Inotropic support or intra-aortic balloon pump device (for weaning the $\mathrm{CPB}$ or in the first 24 hours postoperatively) requirements were also noted.

\section{Statistical Analysis}

Data were analyzed on SPSS 17.0 software (IBM Statistics for Windows version 17, IBM Corporation, Armonk, New York, USA). Distribution of continuous data was assessed using the Kolmogorov-Smirnov test. Quantitative data were expressed as mean \pm standard deviation (SD) and median range (maximumminimum) values in tables. Categorical data were expresses number (n) and percentage. Intergroup comparisons were performed usingrepeated measure ANOVA followed by the Bonferroni test. Time-dependent changes were compared within and between groups. When theMauchly test was significant, normality and equality of variance were not violated, and the Greenhouse-Geisser adjustment was performed to determine the statistical significance of the factors.p values less than 0.05 were regarded as statistically significant.

\section{Results}

The demographic characteristics of the 39 patients taking part in the study were similar between the two groups and are summarized in Table 1. Examination of the study groups in terms of ADA values revealed a significant difference between the two groups 20 min after removal of the cross-clamp $(\mathrm{p}=0.020)$, while other measurements were higher in the DNS group, although the differences were not significant $(\mathrm{p}>0.05$ for all). Comparison of the two groups in terms of time-dependent changes in ADA values revealed that the difference was significant $[\mathrm{F}(3,102)=2.64 \mathrm{p}=0.0432=0.082]$.

XOD values in the DNS were higher than those in the IWBC group at all time points, and significant differences were observed 20 min after removal of the cross-clamp and before cross-clamp removal $(\mathrm{p}=0.023$ and $\mathrm{p}=0.001$, respectively). When values were examined in terms of time, the differences were significant $[\mathrm{F}$ $(3,108)=10.26 \mathrm{p}=0.0172=0.217]$.

CK-MB, SOD, native thiol, total thiol, and thiol disulfide measurement values did not differ between the groups at any time point.No difference was also observed in troponin in the first three measurements, although 
a significant difference was found between the two groups 20 min after cross-clamp removal $\left(4^{\text {th }}\right.$ measurement $\mathrm{p}=0.032$ ).

Analysis of the groups in terms ofPON andarylesterase parametersshowed that both decreased significantly at measurements performed 20 min after cross-clamp removal and before cross-clamp removal, producing a time-dependent difference (PON $[\mathrm{F}(3,108)=3.92 \mathrm{p}=0.0112=0.098]$; arylesterase $[\mathrm{F}(3,108)=3.39 \mathrm{p}=0.021$ $2=0,086])$. However, intergroup comparisons showed that the differences between the groups at the same time points were not significant(Table 3 ).

\section{Discussion}

There are essentially two forms of cardioplegia solution, depending on the content -crystalloidcardioplegia and blood cardioplegia. The aim of all cardioplegia methods, crystalloid-based and blood-based, is to bring about hyperkalemic electromechanical diastolic arrest. The objective is to provide clear vision and surgical fields without motion and to provide optimal protection for the myocardium against ischemic injury. We believe that there have been insufficient studies to comparedel Nidocardioplegia, which has become increasingly used in adult cardiac surgery in recent years, with blood cardioplegia. The present randomized prospective study was intended to compare the biochemical parameters and operative data for the two techniques. Our hypothesis in this study was that conventional blood-based methods enjoy no superiority over del Nidocardioplegia in adult patient groups. The study results indicated that the use of anterograde single-dose DNS in isolated $\mathrm{CABG}$ surgery reduced $\mathrm{CPB}$ and aortic cross-clamp times, yielding similar short-term outcomes to those of a conventional multi-dose IWBC strategy, although no statistically significant reduction was observed. Comparison of the groups' laboratory results showed that at xanthine oxidase measurements were better 20 minutes after cross-clamp placement and immediately before removal, while ADA and troponin measurements were better at measurements after 20 min following cross-clamp removal in the DNS group, the differences being statistically significant(p 0.023, 0.001. 0.020, and 0.032, respectively). Although better results were achieved in the DNS group compared to theIWBC group at other tests, no statistically significant difference was determined. Consistent with the limited number of previous observational studies, our results suggest that del Nidocardioplegia can be safely used in CABG surgery.

Some authors have suggested that the ischemic period and CPB times may be shorter with DNS because this is applied in a single dose, and no additional doses are required for 90 minutes (7). The present research does not allow us to draw any definite conclusion regarding the best timing strategies for additional doses of DNS in CABG surgery. In fact, no consensus exists concerning timings for repetition of DNS. A single dose of DNS is generally sufficient in patients undergoing CABG, and no interruption occurs during surgery. The mean cross-clamp time in the other group was $48.36 \pm 17.26$ minutes, indicating thatcardioplegiawas administered twice on average in each patient. We applied topical hypothermia in all cases in line with our clinical myocardial protection routine. Topical cooling may be beneficial, although some authors have reported no benefit (8).We did not find a significant difference between the two cardioplegia strategies in terms of myocardial injury. XOD,ADA, SOD,PON, arylesterase, native thiol, total thiol, thiol disulfide, troponin-I, and CK-MB levels were similar between the two groups.However, in the DNS group, XOD results were significantly better 20 min after cross-clamp installation and before removal of the cross-clamp, and $\mathrm{ADA}$ and troponin and measurements later than 20 minutes following cross-clamp removal.Consistent with the present study, Yongnan Li et al.'s meta-analysis showedno statistically significant difference between troponin-I, troponin-T, and CK-MB levels in 836 patients (9).

The risk of myocardial dysfunction associated with higher levels of intracellular calcium is greater in older heart surgery patients than in younger individuals (10).DNS has a lower calcium content than standard blood cardioplegia. One recent rat study showedlower intracellular calcium levels in the ischemic period with DNS than with blood cardioplegia (11).As discussed in previous studies, DNS may represent an excellent alternative to conventional techniques in adult cardiac surgery, irrespective of whether patients are low-risk or high-risk (12). The findings of the present study revealed no difference between the two groups in terms ofclinical outcomes including short-term mortality, intensive care unit stay, orinotropic agent requirement. Another advantage in terms of topical coldness is that the crystalloid in the DNS has a ratio of 4:1, and the 
content is at a temperature of $4{ }^{\circ} \mathrm{C}$, compared to $32^{\circ} \mathrm{C}$ for IWBC, which is prepared using warm blood from the CPB circulation. A single dose of cardioplegia was employed in almost all the DNS cases. The single dose of cardioplegia results in less cardioplegia volume and in less hemodilution.

Adenosine deaminase (ADA) regulates purine metabolism through the conversion of adenosine to uric acid (UA). A close association is known to exist between adenosine and UA and cardiovascular events. Tang et al. summarized the impact of ADA on the cardiovascular system in the form of a medical hypotheses involving ADA-mediated inflammatory processes, superoxide radical generation, and the effect of ADA on myocardial ischemia and its potential clinical value (13).Xuan et al. were the first to show an independent correlation between low serum ADA activity levels and CAD occurrence. Those authors reported significant attenuation of serum ADA activity in CAD patients with MI, compared to patients with stable and unstable angina pectoris (14).In the present study, ADA activity in the DNS group was higher than in the IWBC group at all time points.However, the only statistically significant difference was determined 20 min after removal of the cross-clamp $(\mathrm{p}=0.020)$. We think that the value obtained 20 min after removal of the cross-clamp being higher in the DNS group also shows that myocardial protection is not poorer than IWBC.

Paraoxonase-1 is a high-density lipoprotein-associated enzyme exhibitingparaoxonase, arylesterase and dyazoxonase activities. Previous research suggestsa correlation between decreased PON1 activity and with increased oxidative stress, inflammation, and greater atherosclerotic plaque formation, and that it therefore entails an increased risk of cardiovascular disease (15).Vnukov et al.showed that the postoperative period following CABG is associated with paraoxonase suppression, myeloperoxidase activation, and increased arginase activity, nitrite/nitrate levels, and asymmetric dimethylarginine. Those authors also suggested that endothelial dysfunction and a heightened systemic inflammatory response may also be seen (16).In the present study, PON andarylesterase were only lower in the DNS group at the second measurement, while at all other time points they were higher than in the IWBC group, although the difference was not statistically significant. This supports the results in the ADA group. In addition, higher PON at measurements before cross-clamp placement and after cross-clamp removalmay also be interpreted as showing better myocardial protection and a decreased risk of MI. This suggests that PON may be capable of use as a marker in determining cases without MI among coronary artery patients. Identifying patients with low PON values in CAD may allow these to be evaluated as potential candidates for MI and to be followed-up and treated without developing MI.

Thiols (RSH) may be subjected to oxidation reaction via oxidants and create disulfide (RSSR). Under conditions of oxidative stress, the oxidation of cysteine residues can lead to a reversible formation of disulfide bonds between protein thiol groups and low-molecular-mass thiols. These disulfide bonds can also be reduced back to thiol groups. This process thus maintains dynamic thiol-disulfide homeostasis, with itscrucial role in antioxidant protection and detoxification $(17,18)$. No difference was determined between the groups in terms of native thiol, total thiol or thiol disulfide measurements in the present study. These findings also support the idea of good myocardial protection in the DNS group.

The XODand NADPH oxidase systems and the mitochondrial electron transport chain have been implicated in oxidative stress in a number of different organs, such as the intestine, lung, heart, brain, muscle, liver, pancreas, stomach, and kidney (19).XOD results were higher in the DNS group at all time points in the present study, although statistical significance was observed only in the second and third measurements ( $\mathrm{p}$; 0.023 and 0.001 , respectively).

Other researchers have reported successful results with del Nidocardioplegia for high-risk patients with acute ischemia. This strategyis therefore effectivein high-risk patients with acute ischemia, as well as in low-risk patients (20).

\section{Study Limitations}

There are a number of limitations to the present study. First, the relatively low patient number makes it difficult to determine a statistically significant difference for our primary and secondary outcomes. The single-surgeon research design may also lead to patient selection biases, although we endeavored to minimize 
this since the surgeon was not permitted to select the cardioplegia strategy used for each patient. Another limitation is that cardiac functions were not evaluated using postoperativecardiac output monitoring and echocardiograms. The short follow-up timerepresents furtherlimitation in thisand all presently available studies, and is a subject requiring further consideration in the future.

\section{Conclusions}

In conclusion, our clinical and laboratory outcomes in patients undergoing CABG surgery using DNS are quite satisfactory.This is a single-center study which focused on the clinical and laboratory outcomes of the procedure and its use in adult cardiac surgery. Our results are encouraging for further studies. We conclude that single-dose antegrade del Nidocardioplegiarepresents a safe and efficient option for on-pump coronary artery bypass surgery in low-risk patients.

We also think that that PON may be capable of use as a marker in determining cases without MI among coronary artery patients. We think that identifying patients with low PON values in CAD will allow these to be evaluated as potential candidates for MI and to be followed-up and treated without developing MI.

\section{Conflicts of Interest / Funding / Grants}

This project was supported by theKahramanmaraşSütçü İmam University Scientific Research Project (Project no. 2019/6-27M).

\section{References}

1.Kim K, Ball C, Grady P, Mick S. Use of del Nidocardioplegia for adult cardiac surgery at the Cleveland Clinic: perfusion implications. J Extra CorporTechnol2014; 46: 317-323.

2.Ucak HA, Uncu H.Comparison of Del Nido and Intermittent Warm Blood Cardioplegia in Coronary Artery Bypass Grafting Surgery.Ann ThoracCardiovasc Surg. 2019 Feb 20;25(1):39-45. doi: 10.5761/atcs.oa.1800087.

3.O'Donnell C, Wang H, Tran P, Miller S, Shuttleworth P, Boyd JH.Utilization of Del NidoCardioplegia in Adult Coronary Artery Bypass Grafting- A Retrospective Analysis.Circ J. 2019 Jan 25;83(2):342-346. doi: 10.1253/circj. CJ-18-0780.

4.Guajardo Salinas GE, Nutt R, Rodriguez-Araujo G.Del Nidocardioplegia in low risk adults undergoing first time coronary artery bypass surgery.Perfusion. 2017 Jan;32(1):68-73. doi: 10.1177/0267659116661051.

5.Giuisti G. Enzyme activities. In: Method of enzymatic analysis. (Ed. Bergmeyer U), Pp. 1092- 8. GmbltWeinheim, Bergest: VerlogChemie, 1974.

6.Erel O, Neselioglu S. A novel and automated assay for thiol/disulphide homeostasis. ClinBiochem. 2014;47(18):326e332.

7.Timek T, Willekes C, Hulme O, et al. Propensity matched analysis of del Nidocardioplegia in adult coronary artery bypass grafting: initial experience with 100 consecutive patients. Ann ThoracSurg 2016; 101: 2237-41.

8.Baur HR, Peterson TA, Yasmineh WG, et al. Cold potassium cardioplegia versus topical hypothermia and intermittent aortic occlusion for myocardial protection during coronary artery surgery: a randomized clinical study. Ann ThoracSurg 1986; 41: 511-4.

9.Li Y, Lin H, Zhao Y, et al. Del Nidocardioplegia for myocardial protection in adult cardiac surgery: a systematic review and meta-analysis. ASAIO J 2018; 64: 360-7.

10.Shahian DM, O'Brien SM, Filardo G, et al. The society of thoracic surgeons 2008 cardiac surgery risk models: part 1-coronary artery bypass grafting surgery. Ann ThoracSurg 2009; 88: 2-22.

11. O'Blenes SB, Friesen CH, Ali A, et al. Protecting the aged heart during cardiac surgery: the potential benefits of del Nidocardioplegia. J ThoracCardiovascSurg 2011; 141: 762-70. 
12.Guajardo Salinas GE, Nutt R, Rodriguez-Araujo G. Del Nidocardioplegia in low risk adults undergoing first time coronary artery bypass surgery. Perfusion 2017; 32: 68-73.

13.Tang R, Ma C, Dong J, et al . Does adenosine deaminase play a key role in coronary artery disease. Med Hypotheses 2006; 67: 371-374.

14. Xuan C,Tian QW, Zhang SY, Li H, Tian TT, Zhao P, Yue K, Ling YY,He GW, Lun LM. Serum adenosine deaminase activity and coronary artery disease: a retrospective case-control study based on 9929 participants.TherAdv Chronic Dis.2019 Dec 6;10:2040622319891539. doi: 10.1177/2040622319891539.

15.Abello D, Sancho E, Camps J, et al. Exploring the role of paraoxonases in the pathogenesis of coronary artery disease: a systematic review. Int J MolSci2014; 15:20997e21010.

16.Vnukov VV, Sidorov RV, Gvaldin DY, Milyutina NP, Ananyan AA, Pospelov DY, Plotnikov AA, Shlyk IF, Doltmurzieva NS. The role of myeloperoxidase, paraoxonase and nitric oxide system in blood and pericardial fluid in patients with IHD who underwent direct myocardial revascularization.Adv Gerontol.2019;32(1-2):93101.

17.Sanrı Serhat U, Özsin Kaan K, Toktaş F, Balcı Burak A, Üstündă̆ Y, Huysal K, et al. Theeffect of thiol-disulfidehomeostasis in patientsundergoing on-pumpcoronaryartery bypass grafting. TurkGogus Kalp Dama 2019;27:484-492

18.Sivri S, Kasapkara HA, Polat M, Alsancak Y, Durmaz T, Erel Ö, Bozkurt E. Dynamic thiol/disulphide homeostasis and its prognostic value in patients with non-ST elevation-acute coronary syndromes.Kardiol Pol.2018;76(2):426-432. doi: 10.5603/KP. a2017.0208.

19.Wu M.-Y, Yiang G-T, Liao W.-T, Tsai A.P.-Y, Cheng Y.-L, Cheng P.-W, Li C.-Y, Li C.-J. Current Mechanistic Concepts in Ischemia and Reperfusion Injury. Cell PhysiolBiochem2018; 46:16501667.doi:10.1159/000489241.

20. Yerebakan H, Sorabella RA, Najjar M, et al. Del NidoCardioplegia can be safely administered in high-risk coronary artery bypass grafting surgery after acute myocardial infarction: a propensity matched comparison. J CardiothoracSurg2014; 9: 141.

\section{Hosted file}

Consort.docx available at https://authorea.com/users/327205/articles/454836-comparisonof-del-nido-and-intermittent-warm-blood-cardioplegia-in-adult-coronary-artery-bypasssurgery-randomized-prospective-single-center-trial

\section{Hosted file}

Table 1.docx available at https://authorea.com/users/327205/articles/454836-comparisonof-del-nido-and-intermittent-warm-blood-cardioplegia-in-adult-coronary-artery-bypasssurgery-randomized-prospective-single-center-trial

\section{Hosted file}

Table 2.docx available at https://authorea.com/users/327205/articles/454836-comparisonof-del-nido-and-intermittent-warm-blood-cardioplegia-in-adult-coronary-artery-bypasssurgery-randomized-prospective-single-center-trial

\section{Hosted file}

Table 3.docx available at https://authorea.com/users/327205/articles/454836-comparisonof-del-nido-and-intermittent-warm-blood-cardioplegia-in-adult-coronary-artery-bypasssurgery-randomized-prospective-single-center-trial 